Research Article

\title{
Monitoring Sea Ice in Liaodong Bay of Bohai Sea during the Freezing Period of 2017/2018 Using Sentinel-2 Remote Sensing Data
}

\author{
Zhiyong Wang $\mathbb{B}^{1},{ }^{1}$ Peilei Sun, ${ }^{2}$ Lihua Wang, ${ }^{3}$ Mengyue Zhang, ${ }^{1}$ and Zihao Wang ${ }^{1}$ \\ ${ }^{1}$ College of Geodesy and Geomatics, Shandong University of Science and Technology, Qingdao 266590, China \\ ${ }^{2}$ Shenyang Survey and Mapping Research Institute Co. Ltd., Shenyang 110004, China \\ ${ }^{3}$ Changiiang River Scientific Research Institute, Wuhan 430010, China \\ Correspondence should be addressed to Zhiyong Wang; wzywlp@163.com
}

Received 12 March 2021; Revised 10 October 2021; Accepted 12 October 2021; Published 29 October 2021

Academic Editor: Austin Nevin

Copyright ( 2021 Zhiyong Wang et al. This is an open access article distributed under the Creative Commons Attribution License, which permits unrestricted use, distribution, and reproduction in any medium, provided the original work is properly cited.

It is of great significance to monitor sea ice for relieving and preventing sea ice disasters. In this paper, the growth and development of sea ice in Liaodong Bay of Bohai Sea in China were monitored using Sentinel-2 remote sensing data during the freezing period from January to March in 2018. Based on the comprehensive analysis of the spectral characteristics of seawater and sea ice in visible bands, supplemented by the Normalized Difference Snow Index (NDSI) and the Normalized Difference Vegetation Index (NDVI), we proposed a new method based on decision tree classification for extracting sea ice types in Liaodong Bay of Bohai Sea. Using the remote sensing data of eight satellite overpasses acquired from Sentinel-2A/B satellites, the distribution and area of the different sea ice types in Liaodong Bay during the freezing period of 2017/2018 were obtained. Compared with the maximum likelihood (ML) classification method and the support vector machine (SVM) classification method, the proposed method has higher accuracy when discriminating the sea ice types, which proved the new method proposed in this paper is suitable for extracting sea ice types from Sentinel-2 optical remote sensing data in Liaodong Bay. And its classification accuracy reaches $88.05 \%$. The whole process of evolution such as the growth and development of sea ice in Liaodong Bay during the freezing period from January to March in 2018 was monitored. The maximum area of sea ice was detected on 27 January 2018, about $10,187 \mathrm{~km}^{2}$. At last, the quantitative relationship model between the sea ice area and the mean near-surface temperature derived by MODIS data in Liaodong Bay was established. Through research, we found that the mean near-surface temperature was the most important factor for affecting the formation and melt of sea ice in Liaodong Bay.

\section{Introduction}

The Bohai Sea in China is the southernmost frozen sea in the Northern Hemisphere. Affected by the Siberian cold current and the stable cold high-pressure in Mongolia, the sea ice occurs mostly in winter, especially in the north of Bohai Sea, the Liaodong Bay $[1,2]$. Sea ice disaster is a serious natural disaster. The freezing and drifting of sea ice on a large scale are a threat to coastal aquaculture, fishery production, navigation, offshore production operations, and oil-gas exploration, and the resulting economic losses are also serious [3-5]. It is of great significance to effectively monitor the types, the distribution, and the spatiotemporal evolution of sea ice and to analyze the mechanism of its growth, development, and melt for relieving and preventing the sea ice disaster in the Bohai Sea.

Satellite remote sensing technology has the characteristics of economy, timeliness, and large-area simultaneous observation, so it is an important technical means for monitoring the sea ice [2-6]. The satellite remote sensing technology for monitoring sea ice mainly includes microwave radiometer, microwave scatterometer, synthetic aperture radar (SAR), microwave altimeter, hyperspectral remote sensing, and optical remote sensing [2-15]. These technologies have been applied to monitor the sea ice. At present, one of the important parameters of 
monitoring the sea ice is the sea ice types. It is of great significance to accurately discriminate the sea ice types for calculating the area of sea ice, assessing the conditions of sea ice, navigation, marine production operations, and so on.

Many experts and scholars have done a lot of research work in monitoring the sea ice types by satellite remote sensing technology [6-15]. Most of the studies have been carried out on sea ice in polar and high latitudes, and a series of research results have been achieved [7-10]. Dabboor et al. used four-polarized RADARSAT-2 data to classify sea ice of the Arctic region by using a classification method that quantitatively selects the best subset of CP (compact polarimetry) parameters and achieved high classification accuracy [8]. Angelika et al. proposed a semiautomatic classification algorithm and used EM-bird to measure the thickness of sea ice, which improved the accuracy of aerial photographic sea ice classification [9]. Liu et al. used the support vector machine (SVM) method based on texture features and sea ice concentration to classify by using RADARSAT-2 dual-polarized ScanSAR data [10]. A review of the main approaches developed for sea ice classification using satellite imagery was presented [11]. The fully automatic design of a numerically optimized decision tree algorithm and demonstration of its application to sea ice classification from SAR data was introduced [12]. There are relatively few studies on monitoring the sea ice in Bohai Sea. Zhang et al. extracted the polarization scattering characteristics of sea ice using full-polarimetric SAR images and then carried out the research in the classification algorithm of sea ice types by polarimetric SAR [13]. Combining the advantages of support vector machine (SVM) and Markov random field (MRF), Shen et al. proposed a classification system of MRF-v-SVC for sea ice classification of SAR images [14]. Karvonen et al. estimated sea ice parameters including sea ice saturation and sea ice thickness in Bohai Sea, based on thermodynamic sea ice model and SAR, radiometer and other Earth observation data [15]. Microwave sensors, such as SAR, radar altimeter and microwave radiometer, are the main sensors used for monitoring sea ice in polar regions [6-12]. And they are used to monitor the sea ice in the Bohai Sea [13-15], but these sensors have limited capabilities in discriminating sea ice types.

Optical remote sensing has obvious advantages in discriminating the types of terrestrial objects due to its high spatial resolution and abundant spectral information, and it has been applied to monitor the sea ice [16-22]. For example, Shi et al. used MODIS data to obtain the distribution information of sea ice in Bohai Sea $[16,17]$. Su et al. used the method of gray level cooccurrence matrix texture analysis to obtain the distribution of sea ice in Bohai Sea based on the MODIS data [18]. Chen et al. used HJ-1 CCD data and support vector machine classification method to extract sea ice in Liaodong Bay of Bohai Sea [19]. Yan et al. used MODIS data to estimate the amount of sea ice resources in the winter of 2012-2013 [20]. Yan et al. used Geostationary Ocean Color Imager (GOCI) data to monitor the characteristics of the Bohai Sea ice during the winter of 2012-2013, including area and thickness [21].
Through analysis, it can be found that the current research on the sea ice in the Bohai Sea mainly focuses on the distribution, concentration, ice edge, and ice thickness. There were fewer studies on monitoring the sea ice types and the situation of sea ice growth and development in the Bohai Sea. Bohai Sea ice appears every winter in the past 60 years, and this area is an important economic development zone in China and undertaking busy maritime traffic tasks. So it is very important to monitor the sea ice in the Bohai Sea. One of the tasks of monitoring sea ice is to extract the spatial distribution information of sea ice types, which is the sea ice classification mentioned in this paper. Most of the existing studies were based on MODIS data. While the spatial resolution of MODIS data is low, it cannot meet the needs of discriminating sea ice types, and it cannot well be used to analyze the whole process of sea ice growth, development, and melt through the evolution of sea ice types in the Bohai Sea. High-resolution optical satellites (such as SPOT, QuickBird, GeoEye, Worldview, and geostationary satellites) have a long repetition period, which cannot meet the needs of the high temporal resolution in monitoring the growth and development of sea ice. To monitor the growth and development of sea ice in Liaodong Bay of Bohai Sea in detail, it is necessary to find some satellite data with short repetition period and high spatial resolution. With the development of satellite network observation modes, especially in the form of constellation established by multiple satellites, such as the Sentine1-2A/2B optical remote sensing satellite launched by the European Space Agency (ESA) [23-25], its repetition period is greatly shortened, which is conducive to analyze the whole process of sea ice growth and development. In addition, there are many kinds of sea ice, including new ice, white ice, gray-white ice, fast ice, and so on. The difference of spectral reflectance characteristics of different sea ice is much smaller than that of terrestrial objects. It is another difficult problem to be solved to accurately discriminate various sea ice types from similar spectral reflectance. In addition, the typical reflectivities of optical images may be affected by the effect of snow on sea ice and the issue that the waters in Liaodong Bay are turbid and not clear, containing a lot of sediments, complicating optical remote sensing of sea ice in this bay.

Therefore, to solve the problems in monitoring growth and development of sea ice based on remote sensing technique in Liaodong Bay, this paper used Sentine1-2A/2B optical remote sensing data to find a method of extracting sea ice types in Liaodong Bay of Bohai Sea that is suitable for medium-high resolution optical remote sensing satellites. On this basis, the multitemporal data were used to monitor the temporal and spatial evolution of sea ice in Liaodong Bay of Bohai Sea during the freezing period in 2017/2018.

The main sections of this paper are organized as follows. In Section 2, the study area and data are introduced in detail. In Section 3, based on the analysis of the spectral characteristics of sea ice, we propose a new method of extracting the sea ice types of Liaodong Bay that is suitable for mediumhigh resolution optical images. In Section 4, the results are showed. A total of 32 Sentinel-2 optical images from January to March 2018, were classified by decision tree classification. 
And our results were compared with that obtained by the maximum likelihood classification method and support vector machine classification method. In Section 5, the distribution of sea ice types in Liaodong Bay during the freezing period of 2017/2018 is obtained. Then the spatialtemporal evolution of sea ice growth and development in Liaodong Bay was analyzed. The mechanism of growth and development of sea ice in Liaodong Bay was given. Finally, some valuable conclusions drawn from this study are given in Section 6.

\section{Data and Materials}

2.1. Study Area. The Bohai Sea, located in the northeast of China, is the only frozen sea in China. It includes Liaodong Bay, Bohai Bay, and Laizhou Bay. Among them, Liaodong Bay is located in the north of the Bohai Sea. As shown in Figure 1, Liaodong Bay is located at $39-41^{\circ} \mathrm{N}$ and $120.5-122.5^{\circ} \mathrm{E}$. It has the highest latitude and the most serious sea ice disaster area in China. Liaodong Bay freezes every winter in the past 60 years, and the main sea ice types are new ice, white ice, gray-white ice, fast ice.

2.2. Experimental Data. In order to monitor the distribution of sea ice and the whole process of sea ice growth and development in Liaodong Bay of Bohai Sea during the freezing period of 2017/2018, a total of 32 satellite remote sensing images with medium-high resolution acquired from Sentinel-2A/2B were used as the main data source.

The Sentinel-2A satellite was launched on 23 June 2015. It was mainly equipped with the optical sensor called a Multispectral Imager (MSI), including 13 spectral bands, ranging from optical, near infrared to short-wave infrared, with several spatial resolutions (e.g., 10 meters, 20 meters, and 60 meters) [23-25]. The spatial resolution of the panchromatic band is 10 meters, and the resolution of the multispectral band is 20 meters. The central wavelength and resolution of each band of Sentinel-2A/2B is shown in Table 1 . The swath width of the Sentinel-2 satellite image is about $290 \mathrm{~km}$, so it is especially suitable for monitoring the sea ice with a wide range. On 7 March 2017, ESA launched Sentine1-2B, a sister satellite of Sentinel-2A. Its satellite parameters are exactly the same as those of Sentinel-2A. The repetition period of a single satellite is 10 days. In the form of constellation established by two satellites, the period of image acquisition is greatly shortened, and the two satellites can revisit every 5 days in the equatorial region of the Earth. In the case of high latitude regions, the repetition period is only 3 days. The short repetition period of 5 days in Bohai Sea also can provide more data with medium-high resolution when studying the growth and development of sea ice in Liaodong Bay of Bohai Sea. Currently, Sentinel-2A/2B data are officially available for users around the world for free.

We collected 8 satellite overpasses of the Sentine1-2A/2B optical remote sensing data in the study area during the freezing period of 2017/2018, as shown in Table 2. And the cloud covers of all data were less than $10 \%$. There are 3 satellite overpasses data of Sentinel-2A and 5 satellite overpasses data of Sentinel-2B. The swath-width of MSI images from Sentinel-2 is $290 \mathrm{~km}$, but ESA cuts the complete data of each strip into $100 \mathrm{~km}$-sized images and stores them in different files, each of which is less than $1 \mathrm{~GB}$, in order to reduce the amount of data and facilitate data management. So the data of each overpass needs 4 scenes to completely cover Liaodong Bay in Bohai Sea. The details of the experimental data are as follows:

It is not enough to only use the data in the paper to study the growth and evolution of sea ice in Liaodong Bay. However, the focus of this paper is to study an automatic classification method of sea ice based on decision tree, so as to monitor the growth and evolution of sea ice in Liaodong Bay in the winter of 2018.

To make full use of the spectral characteristics of Sentinel-2A/B for discriminating sea ice types, six bands were mainly used, including band $B 2$ (blue), $B 3$ (green), $B 4$ (red), $B 5$ (vegetation red edge), $B 8 A$ (vegetation red edge), and $B 11$ (SWIR, short-wave infrared). The data were uniformly sampled to the pixel size of 20 meters for discriminating the sea ice types.

In addition, in order to derive the near-surface temperature, we used the MODIS L1B (MOD02 $1 \mathrm{~km}$ ) data on the same day as Sentinel-2 data. MODIS data can be obtained at NASA's official website: http://modis.gsfc.nasa. gov/. The imaging time of the MODIS data was all later less than 0.5 hours from that of the Sentinel-2 data. The SRTM (Shuttle Radar Topography Mission) DEM (Digital Elevation Model) and the meteorological data also were used to analyze the growth and development of sea ice in Liaodong Bay. And the SRTM data were downloaded from http://srtm.csi.cgiar.org. The topographic map of study area is shown in Figure 2.

\section{Methods}

At present, there are many classification methods for optical remote sensing images, such as the maximum likelihood classification method, support vector machine (SVM) classification method, and decision tree classification method $[4,22,26]$.

3.1. Technical Method. To meet the needs of discriminating the sea ice types in Liaodong Bay of Bohai Sea, on the basis of analyzing the features of medium-high resolution of Sentinel-2 optical remote sensing image and the spectral characteristics of sea ice, we proposed a method for extracting the sea ice types based on decision tree. Then the whole process of sea ice growth and development in Liaodong Bay of Bohai Sea was monitored by using multitemporal Sentinel-2 optical remote sensing data and the proposed method based on the decision tree. The specific technical method is shown in Figure 3. Firstly, the original Sentinel-2 images should be preprocessed, including radiometric calibration, atmospheric correction, image fusion, and mosaic. Then the training samples of different sea surface objects were selected to analysis spectral characteristics of different sea ice types and to derive the 

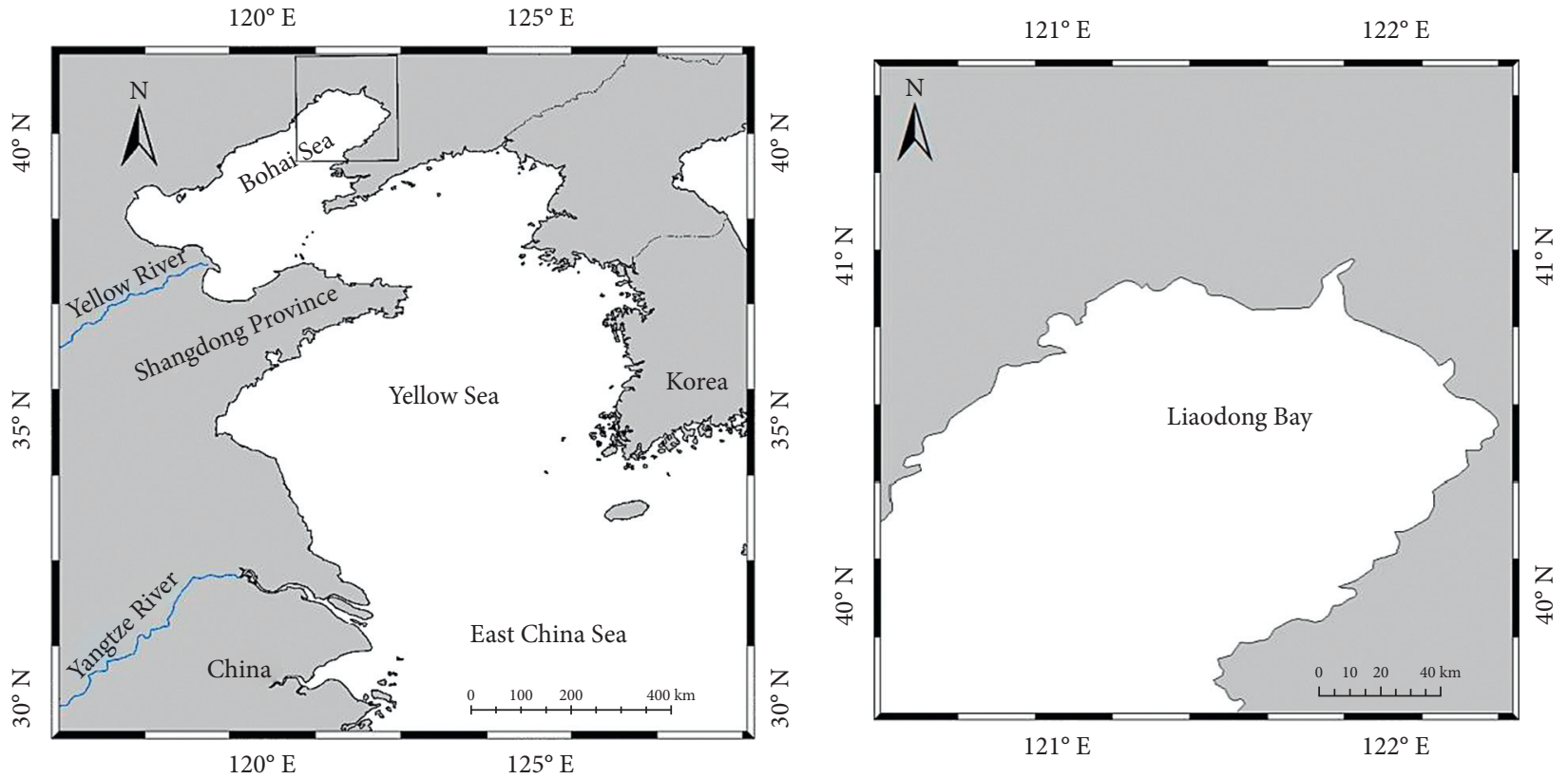

Figure 1: Location map in Liaodong Bay of Bohai Sea in China.

TABLE 1: The central wavelength and resolution of Sentinel-2A/2B.

\begin{tabular}{lcc}
\hline Sentinel-2 bands & Central wavelength $(\mu \mathrm{m})$ & Resolution $(\mathrm{m})$ \\
\hline Band 1 & 0.443 & 60 \\
Band 2 & 0.490 & 10 \\
Band 3 & 0.560 & 10 \\
Band 4 & 0.665 & 10 \\
Band 5 & 0.705 & 20 \\
Band 6 & 0.740 & 20 \\
Band 7 & 0.783 & 20 \\
Band 8 & 0.842 & 10 \\
Band 8A & 0.865 & 20 \\
Band 9 & 0.945 & 60 \\
Band 10 & 1.375 & 60 \\
Band 11 & 1.610 & 20 \\
Band 12 & 2.190 & 20 \\
\hline
\end{tabular}

parameters of NDVI and NDSI. At last, the classification rules suitable for extracting the sea ice types were established by the reflectance differences of various types of sea ice.

3.2. Preprocessing. Firstly, the original Sentinel-2 remote sensing images should be preprocessed, including radiometric calibration, atmospheric correction, image fusion, and mosaic [24]. Radiometric calibration is to convert the grayscale brightness value of an image into absolute radiance brightness value. Atmospheric correction is mainly to eliminate the radiation error caused by atmospheric affect. We used the Sen2Cor plug-in, developed by ESA specifically, to carry out the radiometric calibration and atmospheric correction for the Sentinel-2 MSI images. Due to the wide range of Liaodong Bay in the Bohai Sea, at least 4 scenes of Sentinel-2 remote sensing images were needed to completely cover the whole study area. Therefore, image mosaic was necessary. The color differences between different images need to be dealt with during the mosaic process. The true color composite images of Sentinel-2 data after preprocessing are displayed in Figure 4.

3.3. Establishing the Decision Trees. The decision tree classification algorithm is an inductive learning algorithm, which is based on samples. The algorithm infers classification rules from a set of unordered and irregular samples and establishes a classifier or a prediction model and then classifies the data of unknown categories.

The physical basis of discriminating targets is to distinguish them according to the differences of spectral characteristics between targets. Firstly, sea ice monitoring is actually to discriminate between seawater and sea ice. It is required to analyze the spectral characteristics of seawater and various sea ice types statistically. The spectral curves can be used to analyze the reflectance differences of seawater and various sea ice types in different spectral regions, and then 
TABLE 2: Sentinel-2A/2B experimental data used in this study.

\begin{tabular}{lccccc}
\hline No. & Acquisition date & Sentinel & Orbit number & Sun zenith angle & Local acquisition time \\
\hline 1 & $02 / 01 / 2018^{*}$ & $2 \mathrm{~B}$ & 4,305 & 64.71 & $02: 51: 09.027$ \\
2 & $12 / 01 / 2018$ & $2 \mathrm{~B}$ & 4,448 & 63.72 & $02: 50: 49.027$ \\
3 & $27 / 01 / 2018$ & $2 \mathrm{~A}$ & 13,571 & 61.23 & $02: 49: 51.026$ \\
4 & $01 / 02 / 2018$ & $2 \mathrm{~B}$ & 4,734 & 59.67 & $02: 49: 29.027$ \\
5 & $11 / 02 / 2018$ & $2 \mathrm{~B}$ & 4,877 & 54.28 & $02: 48: 29.027$ \\
6 & $16 / 02 / 2018$ & $2 \mathrm{~A}$ & 13,857 & 53.42 & $02: 48: 01.026$ \\
7 & $21 / 02 / 2018$ & $2 \mathrm{~B}$ & 5,020 & 48.66 & $02: 47: 19.027$ \\
8 & $08 / 03 / 2018$ & $2 \mathrm{~A}$ & 14,143 & $02: 45: 41.026$ \\
\hline
\end{tabular}

${ }^{*}$ Note: day/month/year.

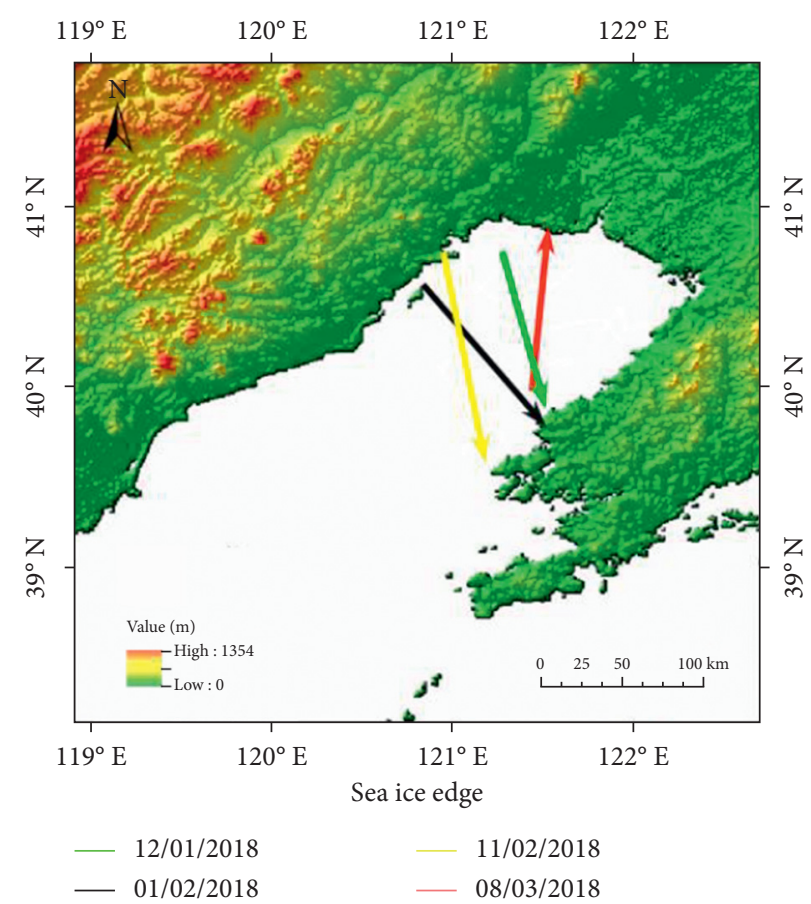

FIgURE 2: Topographic map of study area.

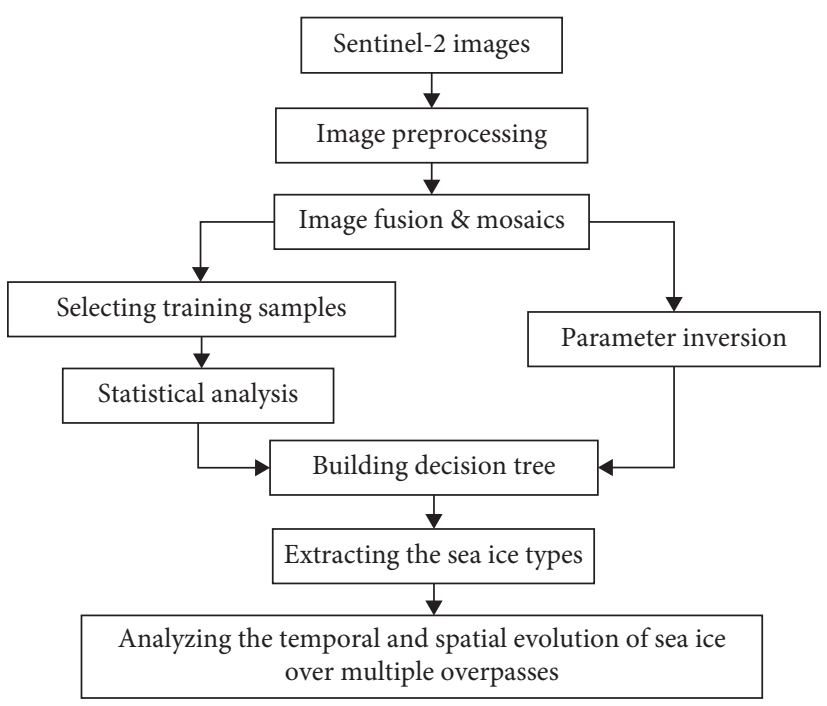

FIGURE 3: Methodology workflow proposed in this paper used for monitoring the growth and development of sea ice in Liaodong Bay. 
$02 / 01 / 2018$

$12 / 01 / 2018$

$121^{\circ} \mathrm{E}$

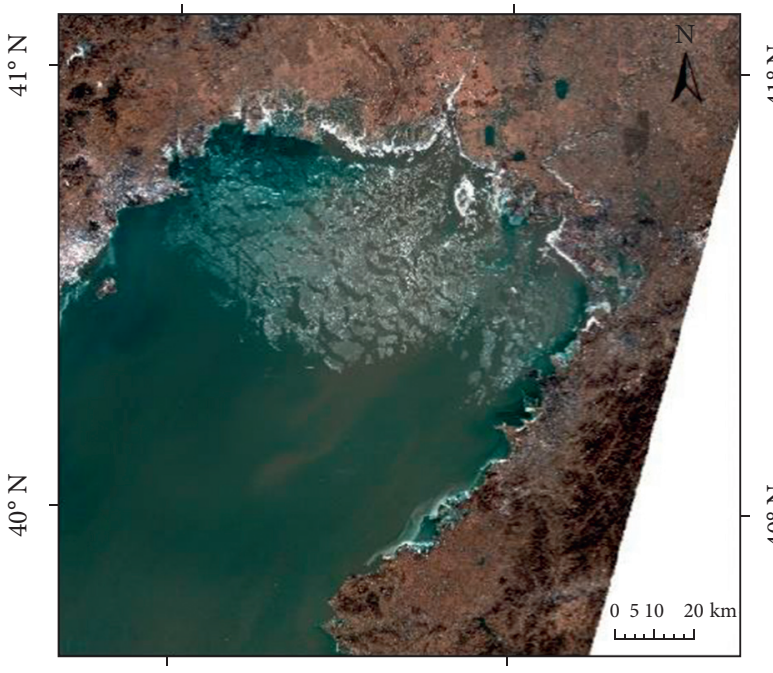

$121^{\circ} \mathrm{E}$

(a)

$27 / 01 / 2018$

$121^{\circ} \mathrm{E}$

\begin{tabular}{l}
$z$ \\
$\circ$ \\
\hdashline
\end{tabular}

$z$
$\vdots$
$\vdots$

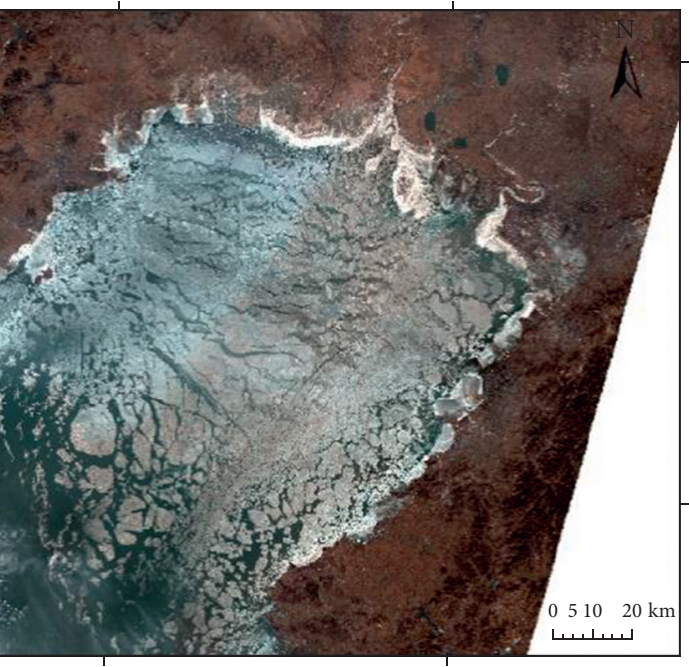

$121^{\circ} \mathrm{E}$ $122^{\circ} \mathrm{E}$

$122^{\circ} \mathrm{E}$

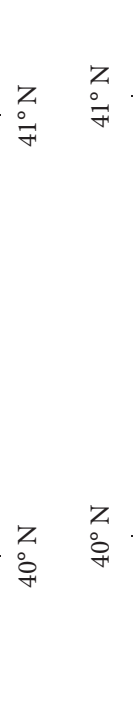

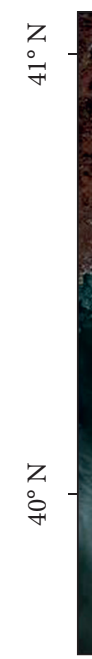

$121^{\circ} \mathrm{E}$

$122^{\circ} \mathrm{E}$

Le

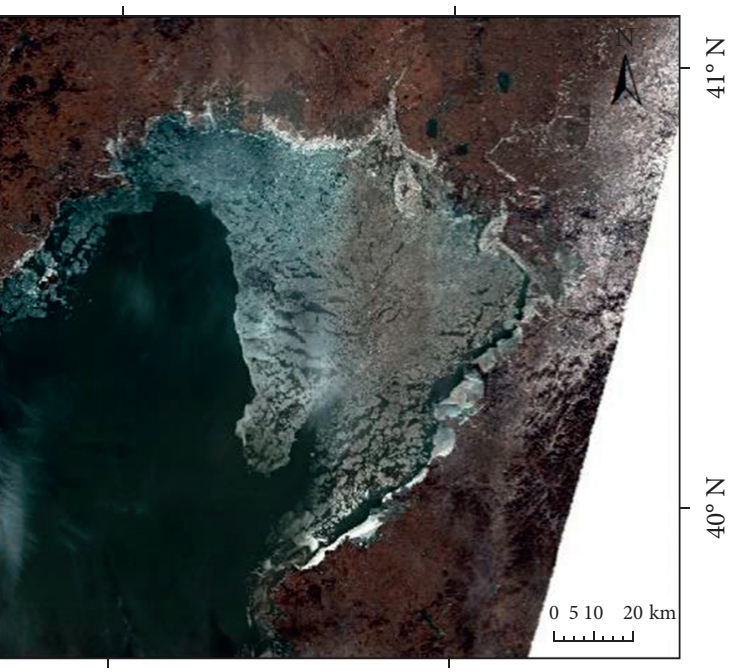

$122^{\circ} \mathrm{E}$

(b)

01/02/2018

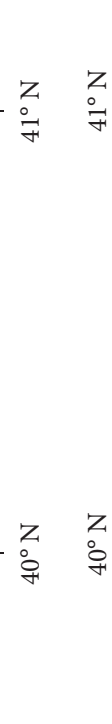

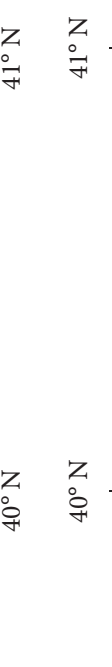

(c) $121^{\circ} \mathrm{E}$

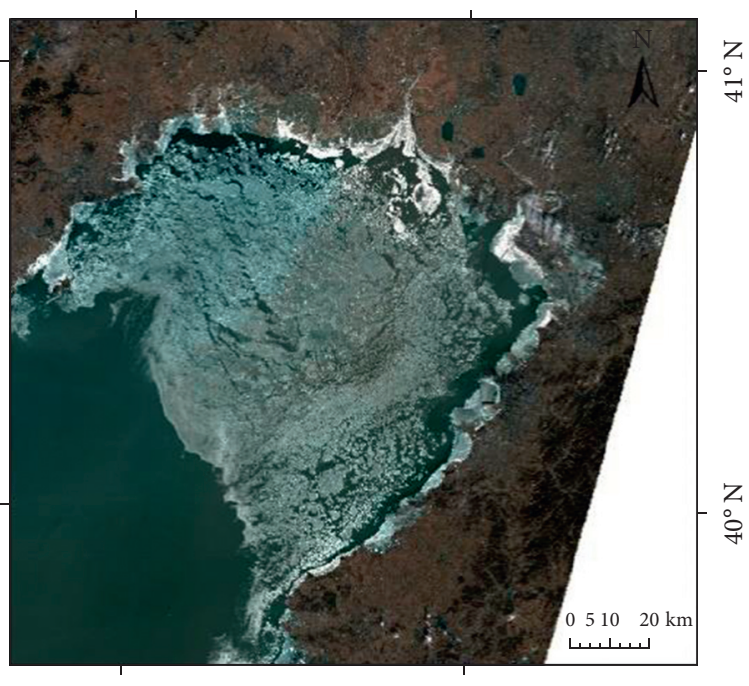

$121^{\circ} \mathrm{E}$ $122^{\circ} \mathrm{E}$

(d)

Figure 4: Continued. 
$122^{\circ} \mathrm{E}$

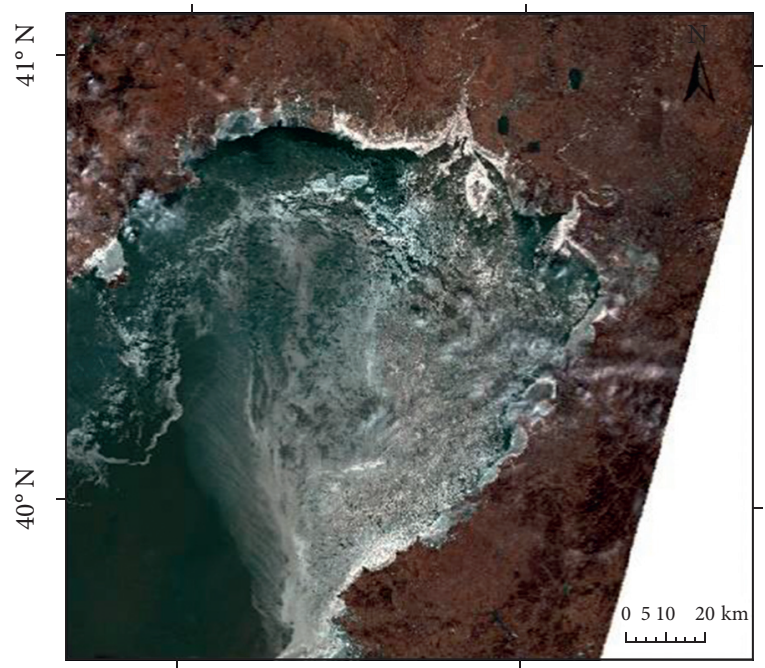

$121^{\circ} \mathrm{E}$

(e)

$21 / 02 / 2018$

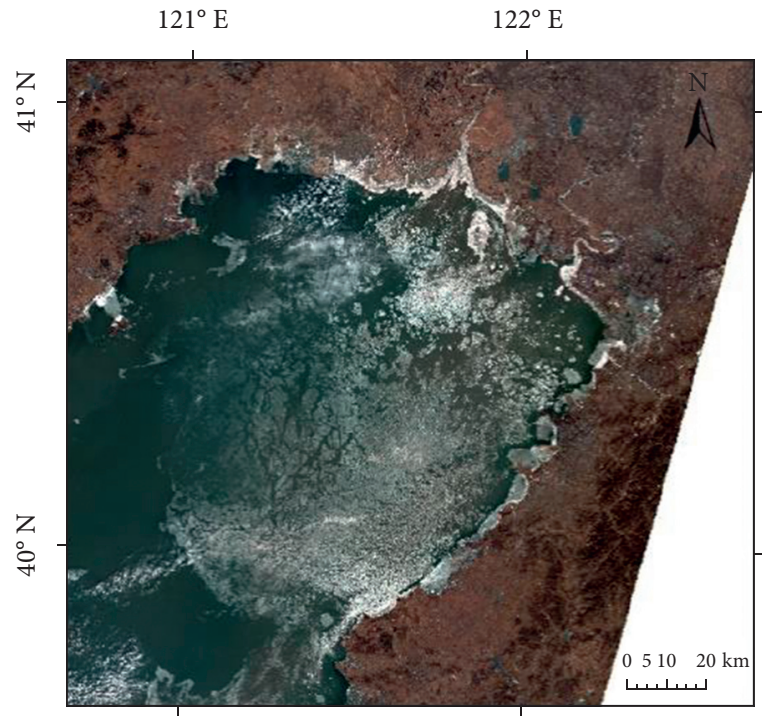

$121^{\circ} \mathrm{E}$ $122^{\circ} \mathrm{E}$

$122^{\circ} \mathrm{E}$

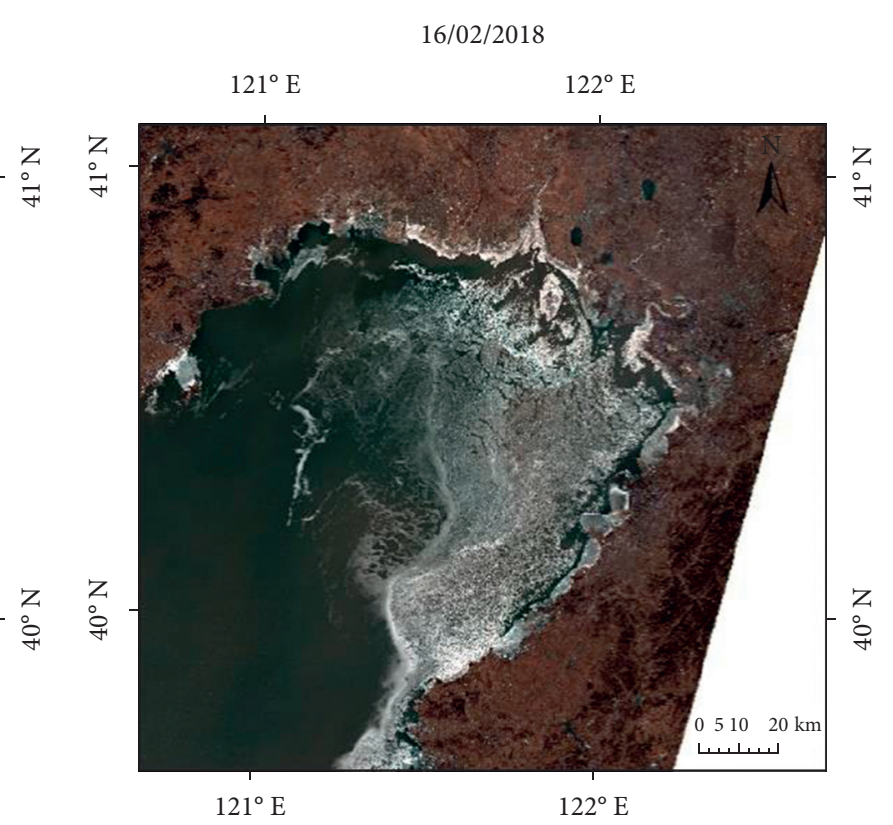

(f)

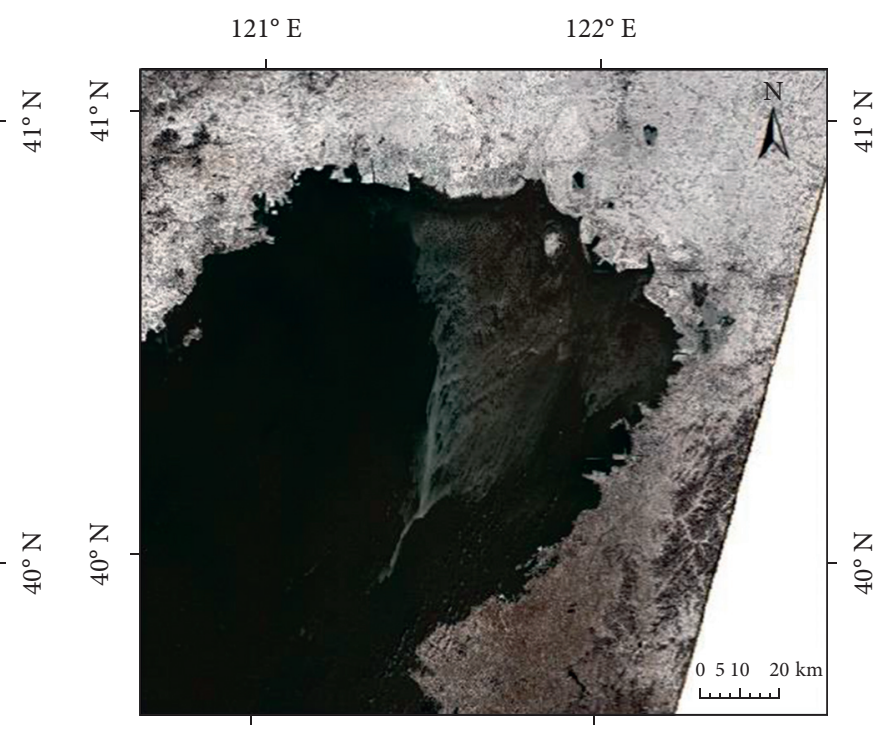

$121^{\circ} \mathrm{E}$

$122^{\circ} \mathrm{E}$

(h)

FIGURE 4: The true color images composited by bands 4,3 , and 2 after preprocessing. (a-h) The true color images in Liaodong Bay on (a) $02 /$ 01/2018, (b) 12/01/2018, (c) 27/01/2018, (d) 01/02/2018, (e) 11/02/2018, (f) $16 / 02 / 2018$, (g) 21/02/2018, and (h) 08/03/2018. The snowfall occurred in Liaodong Bay from 15:00 on 7 March to 2:00 on 8 March 2018. As a result, the color of images acquired on 8 March 2018 is different from the other 7 images.

the classification rules of decision tree classification method can be established to achieve the purpose of sea ice classification [26].

According to the spectral characteristics of sea ice in optical images and the existing research in the Liaodong Bay of Bohai Sea $[1,4,11,18-21]$, the feature types are mainly divided into land area (LA), seawater (SW), fast ice (FI), new ice (NI), white ice (WI), and gray-white ice (GWI).
In order to analyze the spectral characteristics of seawater and various sea ice types, we selected the image acquired on 1 February 2018, in Liaodong Bay as the training sample data set. Through our visual interpretation, multiple ROIs (region of interest) of each type of sea surface target are selected for the samples, and each ROI contains 20-200 pixels. The samples have been indicated in Figure 4(d). A total of 161 training samples were selected, including 50 
seawater training samples, 25 fast ice training samples, 31 new ice training samples, 35 gray-white ice training samples, and 20 white ice training samples.

We should build the rules for the classification of decision tree firstly. As shown in Figure 5, the reflectivity of seawater in each band is low, while the difference between sea ice and seawater in band $B 2$ and band $B 5$ is obvious. So the band $B 2$ and band $B 5$ were selected to discriminate between seawater and sea ice.

We used the Normalized Difference Snow Index (NDSI) to extract snow information [27]. It mainly showed that snow has higher reflectivity in the visible band and lower reflectivity in the short-wave infrared band. We found that characteristics of sea ice are similar to snow. Therefore, NDSI is regarded as important auxiliary information to discriminate the sea ice types. The formula of NDSI is as follows:

$$
\mathrm{NDSI}=\frac{(B 3-B 11)}{(B 3+B 11)},
$$

where $B 3$ represents the green band and $B 11$ represents the short-wave infrared band of Sentinel-2A/B.

Taking band $B 2$ and band $B 5$ as the characteristic bands and using NDSI as the characteristic parameter, the appropriate classification rules were established to monitor the sea ice. Through a lot of experimental analysis, we found that the threshold of the reflectivity of $B 2$ band for discriminating seawater and sea ice is 0.065 , that is, less than 0.065 for seawater and greater than 0.065 for sea ice. The reflectivity of band $B 5$ has a threshold of 0.08 , which is less than 0.08 for seawater and greater than 0.08 for sea ice. The two thresholds are very close to the typical albedo value of open sea water in existing literature [28]. The albedo threshold of band B5 is larger than that of band $B 2$, which may be due to sediment. The Normalized Difference Snow Index (NDSI) has a threshold of 0.65 , which is less than 0.65 for seawater and greater than 0.65 for sea ice. On the basis of sea ice monitoring, the spectral reflectance characteristics of various sea ice types are further explored to achieve sea ice classification.

As can be seen from Figure 6, the reflectivity of fast ice and white ice in each band is high, and the spectral characteristics are not different, so it is difficult to discriminate them only by spectral differences. It is found that the Normalized Difference Vegetation Index (NDVI) has better effect on separating the fast ice from others [29]. The formula for NDVI is as follows:

$$
\mathrm{NDVI}=\frac{(B 8 A-B 4)}{(B 8 A+B 4)},
$$

where $B 4$ represents the red band and $B 8 A$ represents the near infrared band of Sentinel-2A/B. If the value of NDVI is greater than -0.09 , it is classified into fast ice.

After repeated classification experiments and spectral analysis, the optimal threshold of decision tree classification model can be selected. The reflectivity of new ice in band $B 8 A$ is lower than 0.07 , so a classification rule of decision tree is established to discriminate new ice by band $B 8 A$. It can be seen from Figure 6 that the reflectivity of white ice is the most different from that of gray-white ice in the band $B 5$. Therefore, the band $B 5$ is selected as the basis for discriminating white ice from gray-white ice. The threshold value is 0.31 ; that is, the reflectivity of gray-white ice is less than 0.31 , and the reflectivity of white ice is greater than 0.31 .

According to the above analysis of reflectance characteristics, the classification rules suitable for extracting the sea ice types were established by the reflectance differences of various sea ices in each band. Finally, the decision tree classification model for discriminating the sea ice types was generated. The final decision tree of the classification model was established as shown in Figure 7.

\section{Results}

4.1. Classification Results. Based on the proposed method for discriminating the sea ice types, the multiple satellite overpasses data during the freezing period of 2017/2018 in Liaodong Bay were used for classification. The classification results are displayed in Figure 8.

4.2. Comparison of Different Methods. In order to evaluate the effectiveness of the proposed method based on decision tree for sea ice classification, our result of the proposed method was compared with those of maximum likelihood (ML) classification method and support vector machine (SVM) classification method. The images acquired from 11 February 2019, were selected to evaluate the classification accuracy. The ML classification method and SVM classification method are carried out in the same samples. The results of maximum likelihood classification method and support vector machine classification method are shown in Figures 9(a) and 9(b), respectively. The result of the proposed method is shown in Figure 9(c).

By comparing the classification results with the original optical images, we found the distribution of sea ice edge from the proposed method based on decision tree in this paper was clear and basically consistent with the actual distribution. At the same time, it could be seen that the degree with which different ice types are discriminated from each other was also relatively large, and the outline of fast ice around the island and the coastline was substantially the same as that of the original image. In addition, the outline of the white ice in the middle part of the ice region was also better displayed. In terms of efficiency, the proposed method based on decision tree was far superior to the other two algorithms, and it was an effective way to achieve the sea ice classification.

For the images acquired from 11 February 2018, a total of 500 validation samples were generated randomly using the sea ice type maps obtained by different classification methods. Then 500 samples were interpreted by manual visual interpretation. Combining the original optical image, the classification accuracies of various sea ice types were calculated which were determined by the proposed method, the maximum likelihood classification method, and the support vector machine classification method, as shown in Table 3. 




Figure 5: Comparison of reflectivity in different bands of seawater and sea ice.

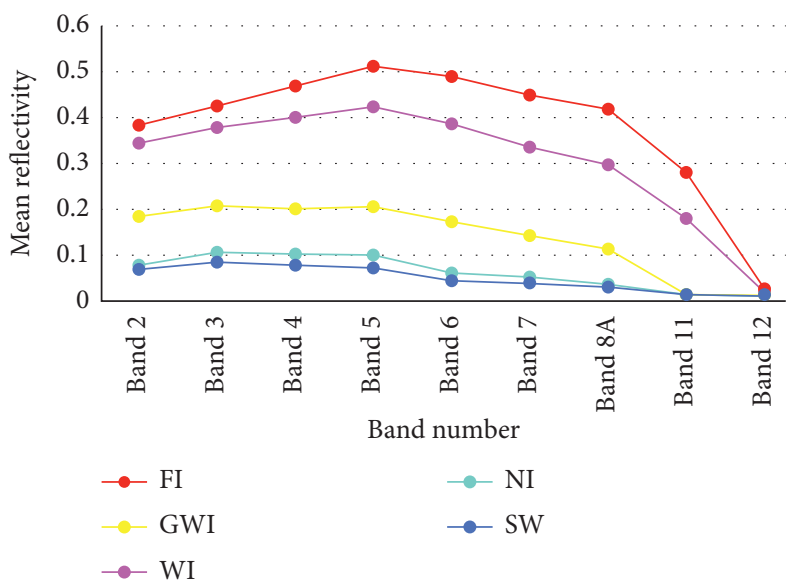

Figure 6: The mean reflectivity of different ice types in different bands of Sentinel-2A/B.

From Table 3, we can see that the classification accuracies of these methods are between $74.30 \%$ and $88.05 \%$. The classification accuracy of the proposed method is higher than the other two methods. And its classification accuracy reaches $88.05 \%$, which indicates that the proposed method is suitable for Sentinel-2 optical remote sensing data to discriminate the sea ice types in Liaodong Bay of Bohai Sea.

\section{Discussion}

5.1. Spatiotemporal Evolution of Sea Ice of Liaodong Bay. The spatiotemporal evolution analysis of sea ice growth, development, and melt was carried out based on the classification results from 2 January to 8 March 2018 (Figure 8). The area of sea ice and its proportion in seawater was calculated, as shown in Table 4.

According to Table 4 and Figure 10, the evolution trend of sea ice in Liaodong Bay was as follows: with the decrease of near-surface temperature, the thickness of sea ice gradually increased. The types of sea ice gradually developed from new ice to gray-white ice and white ice. And the distribution area of sea ice gradually expanded. On 27 January 2018, the sea ice area reached the maximum, which was about $10,187 \mathrm{~km}^{2}$, covering almost the whole Liaodong
Bay. Then, as the near-surface temperature rose, the sea ice slowly melted. And the sea ice area gradually decreased until it finally disappeared completely.

5.2. The Mechanisms of Growth and Development of Sea Ice in Liaodong Bay. The near-surface temperature was derived using the method introduced in reference [30,31] through the MODIS L1B (MOD02 $1 \mathrm{~km}$ ) data of different time. Since the interval of imaging time between MODIS and Sentinel-2 did not exceed half an hour, it could approximately be considered that the mean near-surface temperature derived from the MODIS data represented that of Sentinel-2 imaging. In addition, according to the weather forecast information issued by China Meteorological Administration, the maximum and minimum temperatures in the study area were counted, as shown in Figure 11. It showed the mean near-surface temperature, minimum temperature, and maximum temperature at the eight satellite overpasses in Liaodong Bay during the freezing period in 2017/2018.

According to the distribution of sea ice of Liaodong Bay during the freezing period in 2017/2018, the growth and development of sea ice in Liaodong Bay was mainly affected by the mean near-surface temperature from the MODIS data. 


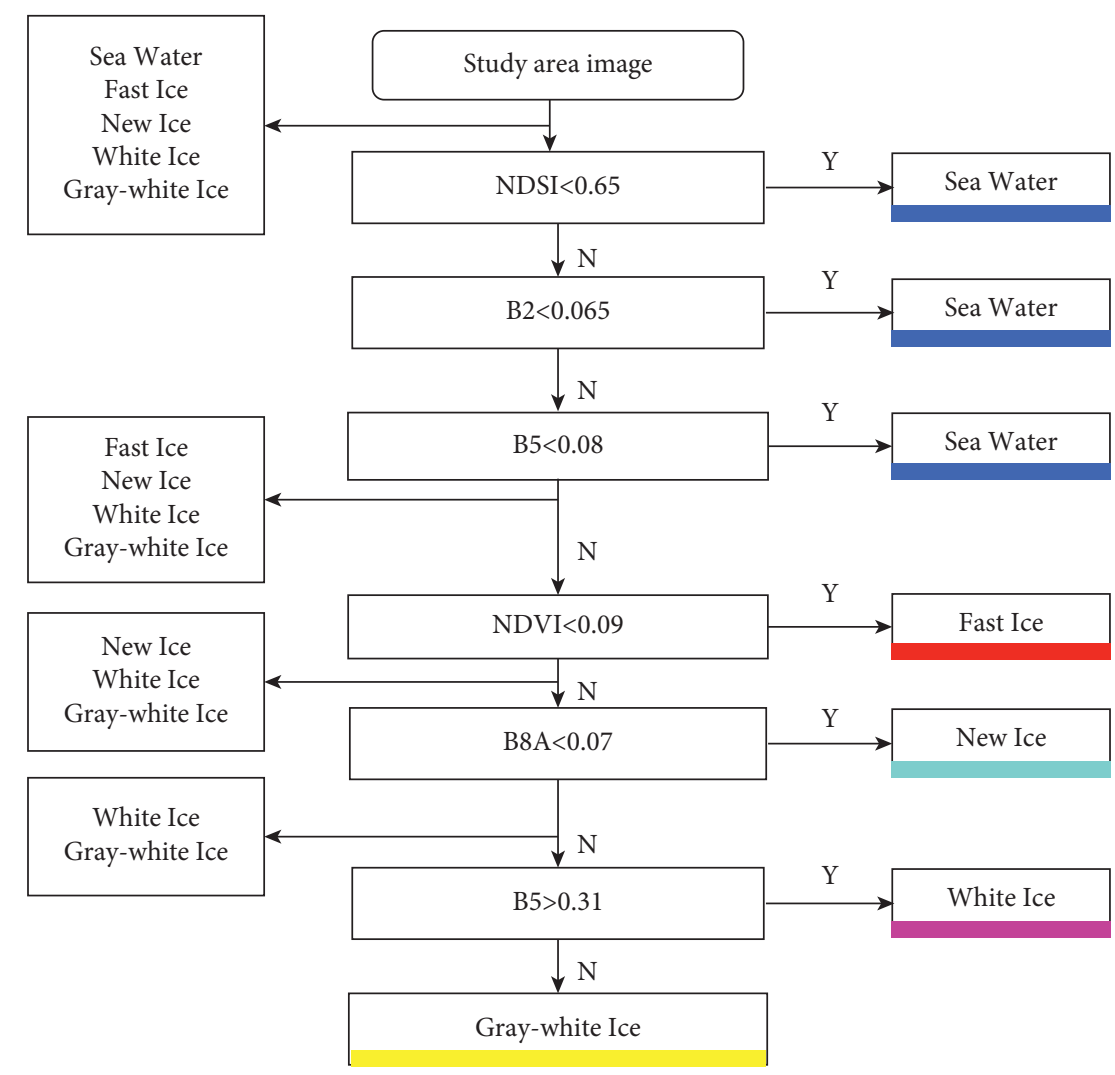

FIGURE 7: A decision tree model for discriminating the sea ice types in Liaodong Bay. The boxes on the left hand side denote the surface types still included in the image before the next classification step.

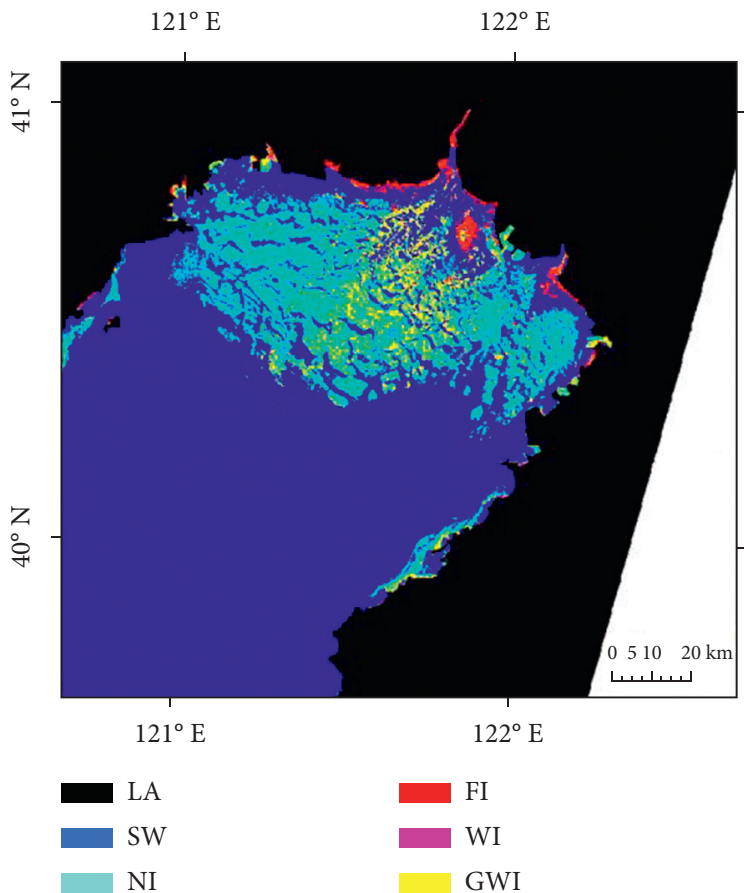

(a)

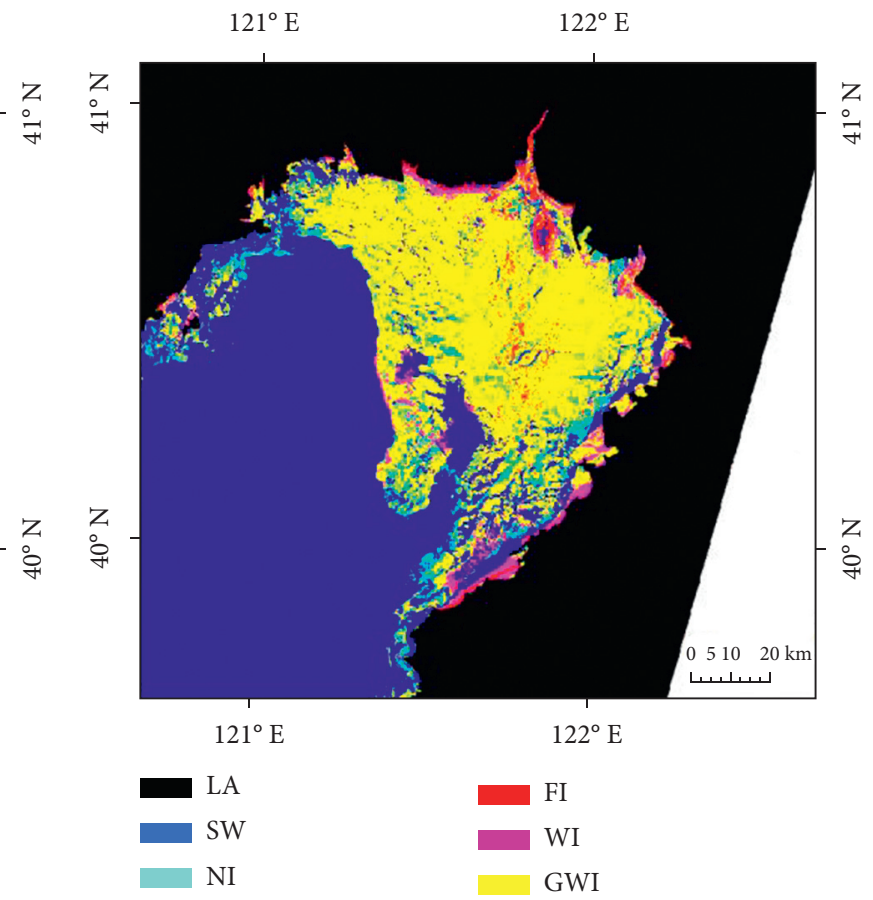

(b)

Figure 8: Continued. 
$121^{\circ} \mathrm{E}$

\begin{tabular}{l}
$Z$ \\
\hdashline \\
\hdashline \\
\hdashline
\end{tabular}

Z

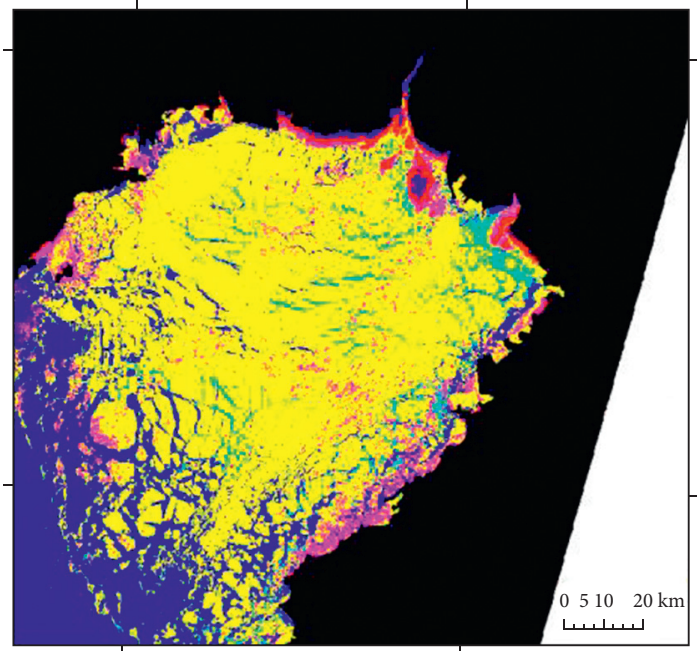

$121^{\circ} \mathrm{E}$

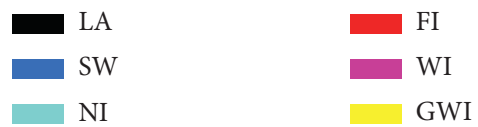

$121^{\circ} \mathrm{E}$

\begin{tabular}{l}
$z$ \\
$\vdots$ \\
\hdashline
\end{tabular}

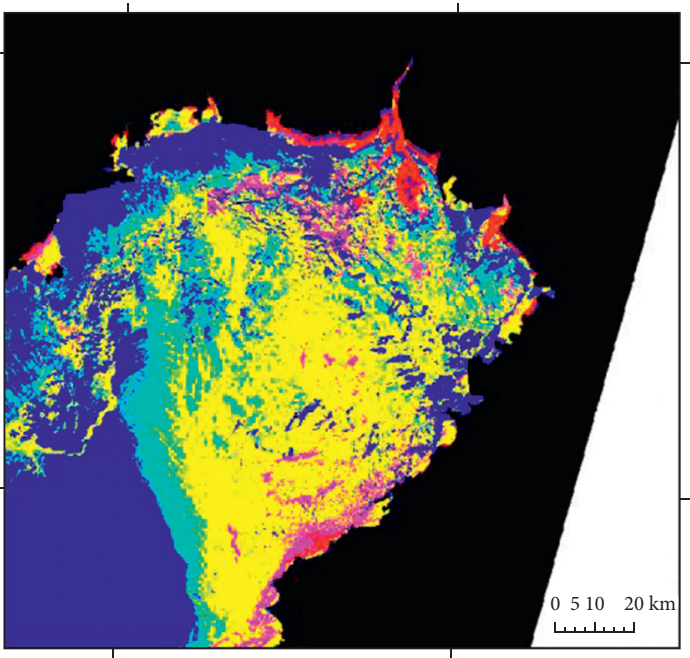

$121^{\circ} \mathrm{E}$

LA

SW

NI (c)

$122^{\circ} \mathrm{E}$

$22^{\circ} \mathrm{E}$

$122^{\circ} \mathrm{E}$

$122^{\circ} \mathrm{E}$

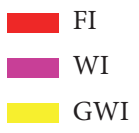

(e) $121^{\circ} \mathrm{E}$

$122^{\circ} \mathrm{E}$
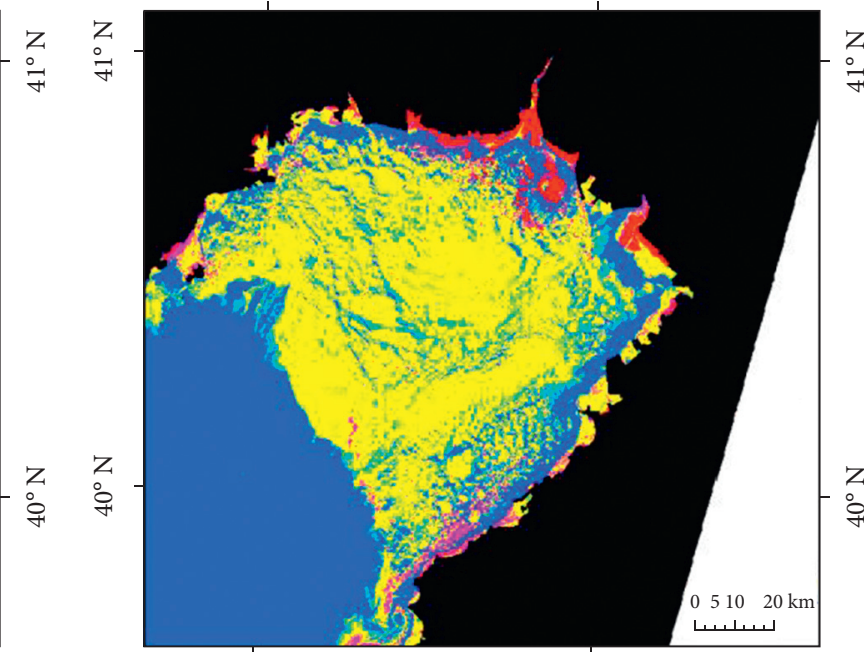

$121^{\circ} \mathrm{E}$

$122^{\circ} \mathrm{E}$

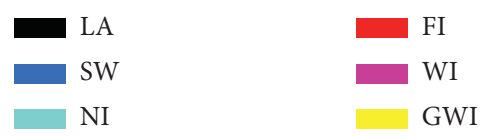

(d)

$121^{\circ} \mathrm{E}$

$122^{\circ} \mathrm{E}$


$121^{\circ} \mathrm{E}$

$122^{\circ} \mathrm{E}$

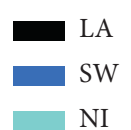

FI

WI

(f)

FIgure 8: Continued. 
$121^{\circ} \mathrm{E}$

\begin{tabular}{l}
$Z$ \\
\hdashline \\
\hdashline
\end{tabular}

요

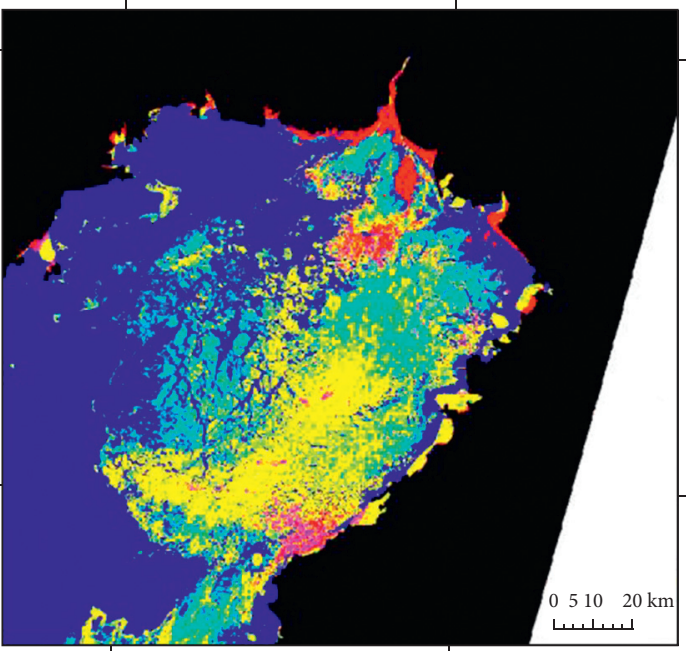

$121^{\circ} \mathrm{E}$

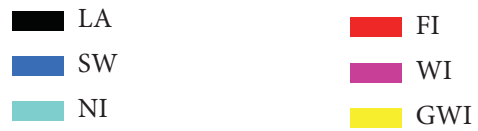

(g) $121^{\circ} \mathrm{E}$

$122^{\circ} \mathrm{E}$


$121^{\circ} \mathrm{E}$

$122^{\circ} \mathrm{E}$

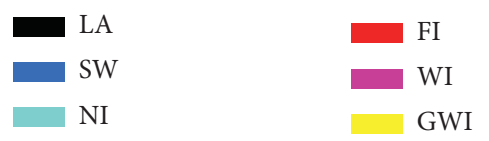

(h)

Figure 8: Distribution maps of sea ice types in Liaodong Bay during the freezing period in 2017/2018. (a-h) The distribution maps of sea ice types in Liaodong Bay on (a) 02/01/2018, (b) 12/01/2018, (c) 27/01/2018, (d) 01/02/2018, (e) 11/02/2018, (f) 16/02/2018, (g) 21/02/2018, and (h) $08 / 03 / 2018$.

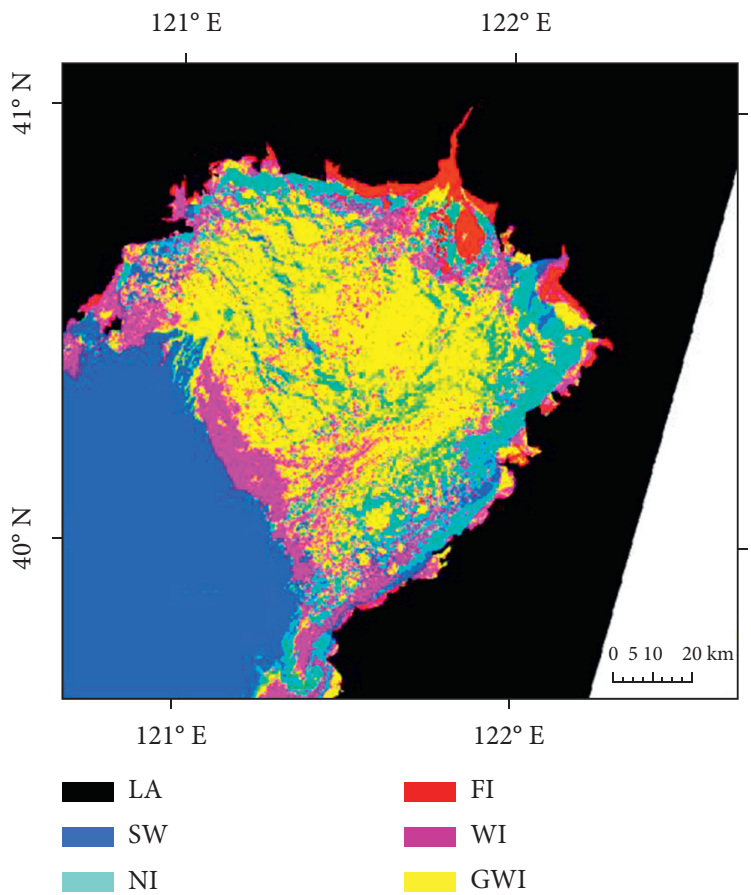

(a)

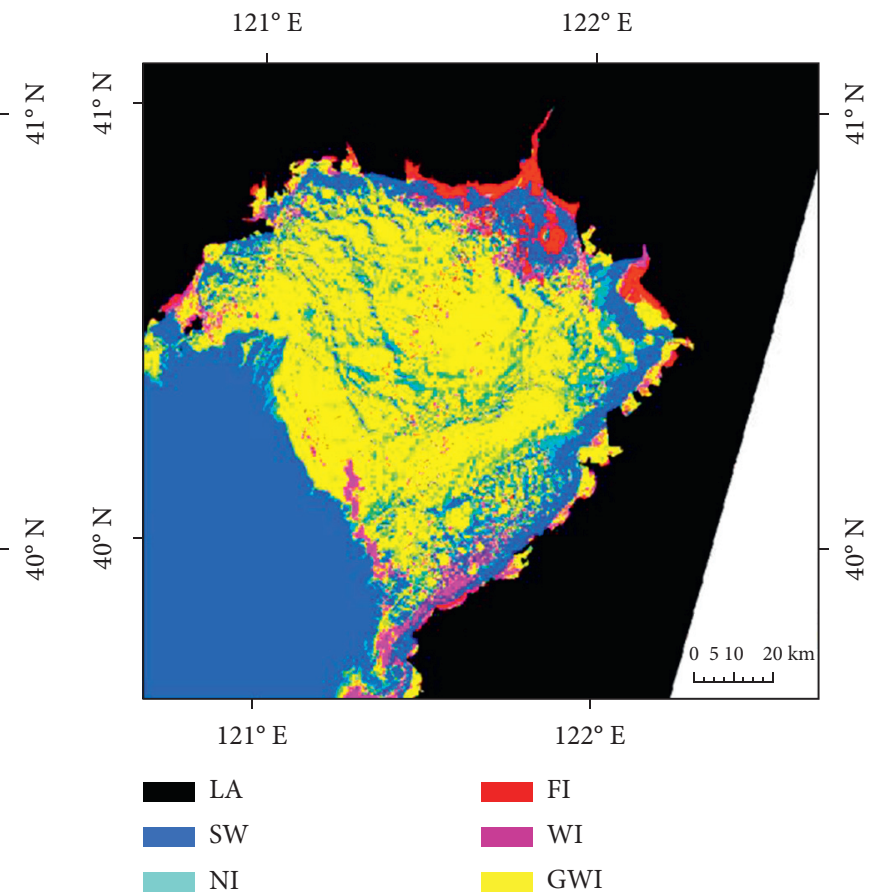

(b)

Figure 9: Continued. 


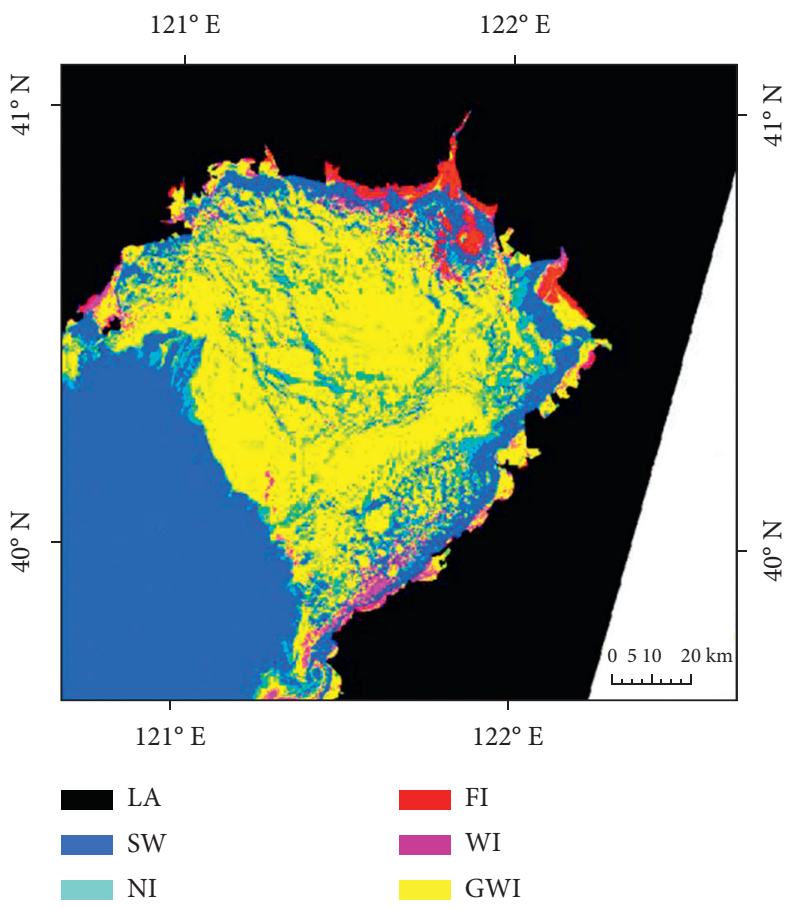

(c)

FIGURE 9: Comparison of sea ice classification results by different methods for the images acquired from 11 February 2019. (a) Classification result of maximum likelihood method; (b) classification result of support vector machine method; (c) classification result of our method.

TABLE 3: Classification accuracy statistics compared with ML and SVM.

\begin{tabular}{lccccccc}
\hline & Seawater (\%) & New ice (\%) & Gray-white ice (\%) & White ice (\%) & Fast ice (\%) & Total accuracy (\%) & Kappa coefficient \\
\hline New method & 97.96 & 93.81 & 76.61 & 83.91 & 90.63 & 88.05 & 0.851 \\
ML & 77.55 & 50.52 & 60.48 & 93.1 & 95.83 & 74.30 & 0.679 \\
SVM & 96.94 & 75.26 & 76.61 & 82.76 & 93.75 & 84.66 & 0.808 \\
\hline
\end{tabular}

TABLE 4: Statistical analysis of the area and the proportion of various sea ice types in Liaodong Bay during the freezing period in 2017/2018.

\begin{tabular}{|c|c|c|c|c|c|c|c|c|c|c|}
\hline \multirow[b]{2}{*}{ Date } & \multicolumn{2}{|c|}{ Seawater } & \multicolumn{2}{|c|}{ New ice } & \multicolumn{2}{|c|}{ Gray-white ice } & \multicolumn{2}{|c|}{ White ice } & \multicolumn{2}{|c|}{ Fast ice } \\
\hline & $\begin{array}{c}\text { Area } \\
\left(\mathrm{km}^{2}\right)\end{array}$ & $\begin{array}{c}\text { Proportion } \\
(\%)\end{array}$ & $\begin{array}{c}\text { Area } \\
\left(\mathrm{km}^{2}\right)\end{array}$ & $\begin{array}{c}\text { Proportion } \\
(\%)\end{array}$ & $\begin{array}{l}\text { Area } \\
\left(\mathrm{km}^{2}\right)\end{array}$ & $\begin{array}{c}\text { Proportion } \\
(\%)\end{array}$ & $\begin{array}{c}\text { Area } \\
\left(\mathrm{km}^{2}\right)\end{array}$ & $\begin{array}{c}\text { Proportion } \\
(\%)\end{array}$ & $\begin{array}{c}\text { Area } \\
\left(\mathrm{km}^{2}\right)\end{array}$ & $\begin{array}{c}\text { Proportion } \\
\text { (\%) }\end{array}$ \\
\hline $\begin{array}{l}02 / 01 / \\
2018\end{array}$ & 10,379 & 76.1 & 2,809 & 20.6 & 273 & 2.0 & 32 & 0.2 & 149 & 1.1 \\
\hline $\begin{array}{l}12 / 01 / \\
2018\end{array}$ & 8,247 & 60.4 & 642 & 4.7 & 4,059 & 29.8 & 474 & 3.5 & 220 & 1.6 \\
\hline $\begin{array}{l}27 / 01 / \\
2018\end{array}$ & 3,454 & 25.3 & 1,141 & 8.4 & 6,098 & 44.7 & 2,510 & 18.4 & 439 & 3.2 \\
\hline $\begin{array}{l}01 / 02 / \\
2018\end{array}$ & 6,481 & 47.5 & 345 & 2.5 & 6,078 & 44.6 & 406 & 3.0 & 332 & 2.4 \\
\hline $\begin{array}{l}11 / 02 / \\
2018\end{array}$ & 4,455 & 32.7 & 2,699 & 19.8 & 5,337 & 39.1 & 726 & 5.3 & 426 & 3.1 \\
\hline $\begin{array}{l}16 / 02 / \\
2018\end{array}$ & 8,230 & 60.3 & 1,034 & 7.6 & 3,345 & 24.5 & 630 & 4.6 & 403 & 3.0 \\
\hline $\begin{array}{l}21 / 02 / \\
2018\end{array}$ & 7,798 & 57.1 & 1,964 & 14.4 & 3,229 & 23.7 & 245 & 1.8 & 406 & 3.0 \\
\hline $\begin{array}{l}08 / 03 / \\
2018\end{array}$ & 10,868 & 79.7 & 670 & 4.9 & 1,883 & 13.8 & 92 & 0.7 & 129 & 0.9 \\
\hline
\end{tabular}




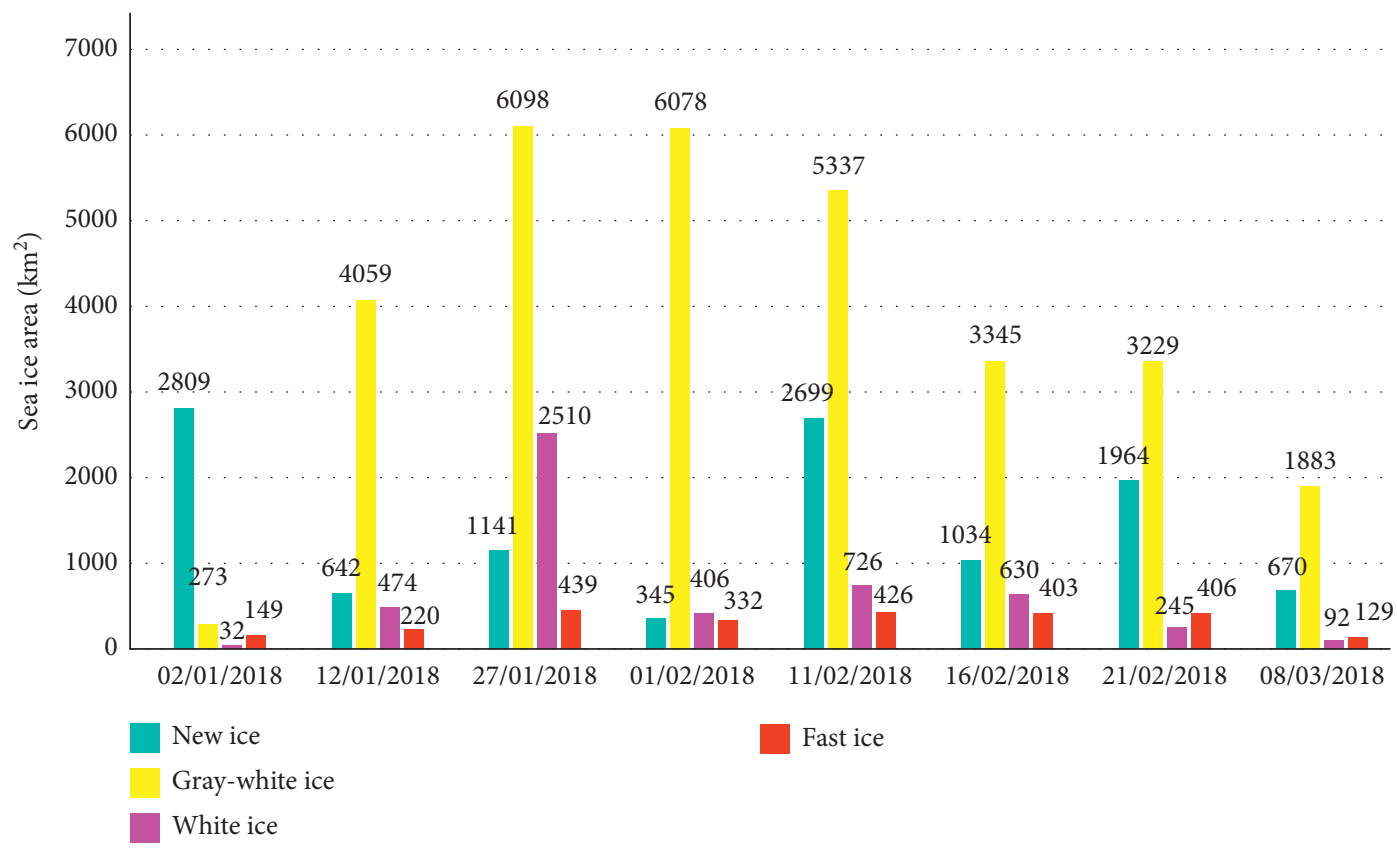

FIGURE 10: The area statistics of various sea ice types in Liaodong Bay during the freezing period in 2017/2018.

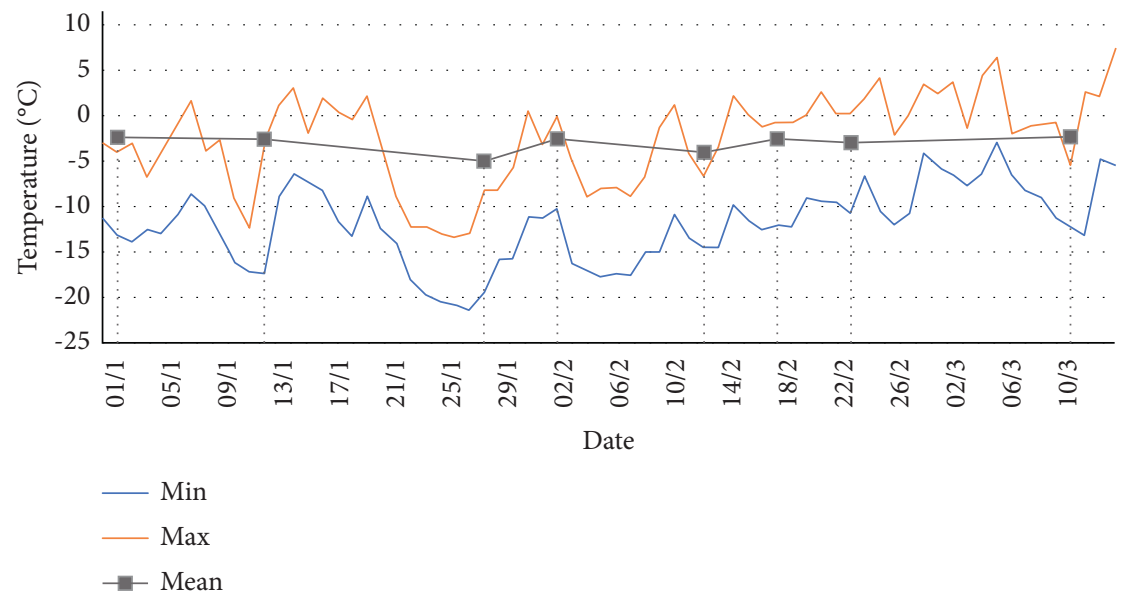

FIgURE 11: Trend of temperature in Liaodong Bay during the freezing period in 2017/2018. The mean near-surface temperatures were derived from the MODIS data. The maximum and minimum temperatures were obtained according to the weather forecast information issued by China Meteorological Administration. The vertical dashed lines represent the acquisition times which the Sentinel-2 images acquired.

When the mean near-surface temperature decreases to $0^{\circ} \mathrm{C}$, it begins to freeze in Liaodong Bay. The sea ice type is mainly new ice, and the thickness of sea ice is thin. As per the further decrease of mean near-surface temperature, the frozen area is expanding, and the sea ice types gradually develop from new ice to gray-white ice and white ice, and the thickness of sea ice gradually increases. If the mean near-surface temperature continues to increase above $0^{\circ} \mathrm{C}$, sea ice will melt and sea ice area will be greatly reduced.
Through linear regression analysis, a quantitative relationship model between the sea ice area and the mean nearsurface temperature derived from the MODIS data was established in Liaodong Bay (as shown in Figure 12). The specific relationship model was as follows:

$$
y=-2063.5 x-160.03
$$

where $y$ is the sea ice area at a certain time (unit: $\mathrm{km}^{2}$ ) and $x$ is the mean near-surface temperature at this time (unit: ${ }^{\circ} \mathrm{C}$ ). 


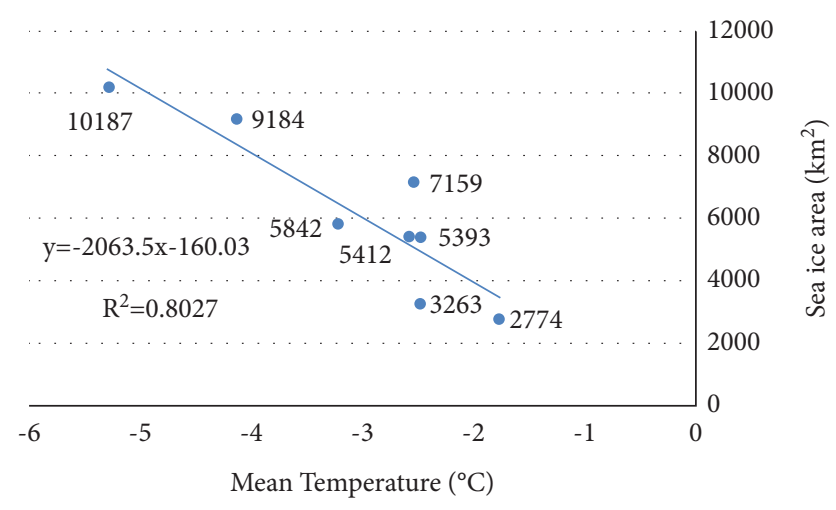

Figure 12: Linear regression analysis of sea ice area and nearsurface temperature in Liaodong Bay during freezing period in $2017 / 2018$

\section{Conclusions}

In this paper, we used a total of 32 Sentinel- 2 optical remote sensing images from January to March 2018 to monitor the sea ice in Liaodong Bay of the Bohai Sea in China. According to the spectral characteristics of sea ice and seawater, the classification rules based on decision tree were established to discriminate the sea ice types at different time. A new method based on decision tree classification for discriminating the sea ice types was proposed. The validity of the new method proposed in this paper was verified. At the same time, combined with the near-surface temperature derived from the MODIS data and meteorological data, the evolution of sea ice distribution and area in Liaodong Bay was analyzed.

Through the study, some valuable conclusions can be drawn as follows:

(1) The new method makes full use of the spectral characteristics, NDSI, and NDVI to discriminate the sea ice types. The classification accuracy of the proposed method was higher than those of the maximum likelihood classification method and the support vector machine classification method. Its classification accuracy reached $88.05 \%$. The test proved that the new method is suitable for Sentinel-2 optical images with medium-high resolution to discriminate the sea ice types.

(2) The growth and development of sea ice in Liaodong Bay during the freezing period in 2017/2018 was analyzed through 8 Sentinel-2 satellite overpasses. As the mean near-surface temperature decreases, the sea ice in Liaodong Bay generally begins to freeze. The maximum area of sea ice was detected on 27 January 2018, about $10,187 \mathrm{~km}^{2}$. The sea types include fast ice, new ice, white ice, and gray-white ice in Liaodong Bay.

(3) The mean near-surface temperature was the most important factor for affecting the formation and melt of sea ice in Liaodong Bay. The quantitative relationship model between sea ice area and mean nearsurface temperature in Liaodong Bay was established, which can provide a theoretical basis for analyzing the growth and development of sea ice in Liaodong Bay.

The results of sea ice classification were affected by image quality and preprocessing. Because it needed 4 scenes of Sentinel-2 images to completely cover the study area, the uniform color processing during image mosaic has a certain influence on the classification accuracy of sea ice. In addition, the selection of training samples would also affect the accuracy of sea ice classification. The sample of the gray-white ice was relatively few, so the classification accuracy of gray-white ice was not very high. The evolution of sea ice was affected by many factors and there were existing randomness and variability.

Although the repetition period of Sentinel-2A/2B satellite network has been reduced to 5 days, the satellite remote sensing data of some satellite overpasses are greatly contaminated by clouds and fogs because of weather reasons, so they cannot be used. This made the data time interval larger in some satellite overpasses, which is not conductive to analyze the growth and development of sea ice in Liaodong Bay in detail. However, using the eight satellite overpasses of Sentinel-2 remote sensing data, the type change of the sea ice growth and development in Liaodong Bay can be basically ascertained. In the next, we will use Sentinel-1 radar satellite data that is not affected by clouds and fogs [32] to analyze the growth and development of sea ice in Liaodong Bay.

\section{Data Availability}

The Sentinel-2 data, MODIS data, and SRTM DEM data can be available for free.

\section{Conflicts of Interest}

The authors declare no conflicts of interest.

\section{Acknowledgments}

Sentinel data were provided by the Copernicus program. The authors thank the USGS (United States Geological Survey) for providing the MODIS data. The authors thank NASA (National Aeronautics and Space Administration) for providing the SRTM DEM data. This research was funded by the National Natural Science Foundation of China (no. 41876202) and the Shandong Province Natural Science Foundation (no. ZR2017MD020).

\section{References}

[1] Z. Xu, Y. Yang, and G. Wang, "Reflex characteristics of sea ice in Liaodong Bay," Spectroscopy and Spectral Analysis, vol. 30, no. 7, pp. 1902-1907, 2010.

[2] S. Yuan, C. Liu, and X. Liu, "Practical model of sea ice thickness of Bohai sea based on MODIS data," Chinese Geographical Science, vol. 28, no. 5, pp. 863-872, 2018.

[3] C. Yue, L. I. Jingkai, and C. Guan, "Surface wave simulation during winter with sea ice in the Bohai Sea," Journal of Oceanology and Limnology, vol. 37, no. 6, pp. 1857-1867, 2018.

[4] X. Zhang, W. Dierking, and J. Zhang, "A polarimetric decomposition method for ice in the Bohai sea using C-band 
PolSAR data," IEEE Journal of Selected Topics in Applied Earth Observations and Remote Sensing, vol. 8, no. 1, pp. 47-66, 2015.

[5] J. Zheng, C. Ke, and Z. Shao, "Winter sea ice albedo variations in the Bohai Sea of China," Acta Oceanologica Sinica, vol. 36, no. 1, pp. 56-63, 2017.

[6] W. Liu, H. Sheng, and X. Zhang, "Sea ice thickness estimation in the Bohai Sea using geostationary ocean color imager data," Acta Oceanologica Sinica, vol. 35, no. 7, pp. 105-112, 2016.

[7] L. K. Soh and C. Tsatsoulis, "Texture analysis of SAR sea ice imagery using gray level co-occurrence matrices," IEEE Transactions on Geoscience and Remote Sensing, vol. 37, no. 2, pp. 780-795, 1999.

[8] M. Dabboor and T. Geldsetzer, "Towards sea ice classification using simulated RADARSAT Constellation Mission compact polarimetric SAR imagery," Remote Sensing of Environment, vol. 140, pp. 189-195, 2014.

[9] H. H. R. Angelika, D. Marie, and B. Justin, "Improved characterisation of sea ice using simultaneous aerial photography and sea ice thickness measurements," Cold Regions Science and Technology, vol. 92, pp. 37-47, 2013.

[10] H. Liu, H. Guo, and L. Zhang, "SVM-based sea ice classification using textural features and concentration from RADARSAT-2 dual-pol ScanSAR data," IEEE Journal of Selected Topics in Applied Earth Observations and Remote Sensing, vol. 8, no. 4, pp. 1601-1613, 2015.

[11] Z. Natalia, S. Vladimir, and B. Irina, "Satellite SAR data-based sea ice classification: an overview," Geosciences, vol. 9, no. 4, p. 152, 2019.

[12] L. Johannes, P. D. Anthony, and D. Wolfgang, “An optimal decision-tree design strategy and its application to sea ice classification from SAR imagery," Remote Sensing, vol. 11, no. 13, Article ID 1574, 2019.

[13] X. Zhang, J. Zhang, and J. Meng, "Study on polarimetric SAR sea ice classification method based on polarimetric scattering characteristics: taking Bohai Sea ice classification as an example," Journal of Oceanography, vol. 35, no. 5, pp. 95-101, 2013.

[14] Y. Shen, W. Lang, and J. Wu, "Classification of SAR sea ice images combined with MRF and v-SVM," Journal of remote sensing, vol. 19, no. 5, pp. 844-855, 2015.

[15] J. Karvonen, L. Shi, B. Cheng, M. Similä, M. Mäkynen, and T. Vihma, "Bohai sea ice parameter estimation based on thermodynamic ice model and earth observation data," Remote Sensing, vol. 9, no. 3, pp. 234-267, 2017.

[16] W. Shi and M. Wang, "Sea ice properties in the Bohai Sea measured by MODIS-Aqua: 1 . Satellite algorithm development," Journal of Marine Systems, vol. 95, no. 1, pp. 32-40, 2012.

[17] W. Shi and M. Wang, "Sea ice properties in the Bohai Sea measured by MODIS-Aqua: 2. Study of sea ice seasonal and interannual variability," Journal of Marine Systems, vol. 95, no. 1, pp. 41-49, 2012.

[18] H. Su, Y. Wang, J. Xiao, and L. Li, "Improving MODIS sea ice detectability using gray level co-occurrence matrix texture analysis method: a case study in the Bohai Sea," ISPRS Journal of Photogrammetry and Remote Sensing, vol. 85, pp. 13-20, 2013.

[19] W. Chen, H. He, and H. Huang, "Study on spatial and temporal distribution of sea ice using HJ-1 CCD data-A case study of Liaodong Bay in Bohai, China," Safety and Environmental Engineering, vol. 21, no. 5, pp. 121-125, 2014.
[20] Y. Yan, L. Xu, and Y. Xu, "The estimation of ice resources in the Bohai Sea based on MODIS Data," Resources Science, vol. 39, no. 11, pp. 2166-2175, 2017.

[21] Y. Yan, K. Huang, D. Shao, Y. Xu, and W. Gu, "Monitoring the characteristics of the Bohai sea ice using high-resolution Geostationary Ocean color imager (GOCI) data," Sustainability, vol. 11, no. 3, p. 777, 2019.

[22] Y. Liu, J. Key, and R. Mahoney, "Sea and freshwater ice concentration from VIIRS on suomi NPP and the future JPSS satellites," Remote Sensing, vol. 8, no. 6, p. 523, 2016.

[23] J. Li and D. Roy, "A global analysis of sentinel-2A, sentinel-2B and landsat- 8 data revisit intervals and implications for terrestrial monitoring," Remote Sensing, vol. 9, no. 9, pp. 902-919, 2017.

[24] N. Pahlevan, S. Sarkar, B. A. Franz, S. V. Balasubramanian, and J. He, "Sentinel-2 MultiSpectral Instrument (MSI) data processing for aquatic science applications: demonstrations and validations," Remote Sensing of Environment, vol. 201, pp. 47-56, 2017.

[25] M. A. Warren, S. G. H. Simis, V. Martinez-Vicente et al., "Assessment of atmospheric correction algorithms for the Sentinel-2A MultiSpectral Imager over coastal and inland waters," Remote Sensing of Environment, vol. 225, pp. 267289, 2019.

[26] X. Guan, Research on Classification Algorithm Based on Decision Tree, Shanxi University, Taiyuan, China, 2006.

[27] D. K. Hall, G. A. Riggs, and V. V. Salomonson, "Development of methods for mapping global snow cover using moderate resolution imaging spectroradiometer data," Remote Sensing of Environment, vol. 54, no. 2, pp. 127-140, 1995.

[28] S. Hua, Y. Wang, and J. Yang, "Monitoring the spatiotemporal evolution of sea ice in the Bohai sea in the 2009-2010 winter combining MODIS and meteorological data," Estuaries and Coasts, vol. 35, pp. 281-291, 2012.

[29] Y. Zhou, C. L. Gong, and Y. Hu, "Extraction of sea ice information in the arctic ocean by using FY-3/MERSI data," Journal of Atmospheric and Environmental Optics, vol. 8, pp. 53-59, 2013.

[30] L. M. McMillin, "Estimation of sea surface temperatures from two infrared window measurements with different absorption," Journal of Geophysical Research, vol. 80, no. 36, pp. 5113-5117, 1975.

[31] P. J. Minnett, A. Alvera-Azcárate, T. M. Chin et al., "Half a century of satellite remote sensing of sea-surface temperature," Remote Sensing of Environment, vol. 233, Article ID 111366, 2019.

[32] Z. Wang, J. Liu, J. Wang et al., "Resolving and analyzing landfast ice deformation by InSAR technology combined with Sentinel-1A ascending and descending orbits data," Sensors, vol. 20, no. 22, Article ID 6561, 2020. 